\title{
Nonconvexity of the set of hypergraph degree sequences
}

\author{
Ricky Ini Liu \\ University of Michigan \\ Ann Arbor, Michigan, U.S.A. \\ riliu@umich.edu \\ Submitted: Sep 17, 2012; Accepted: Jan 17, 2013; Published: Jan 29, 2013 \\ Mathematics Subject Classifications: 05C65, 05C07

\begin{abstract}
It is well known that the set of possible degree sequences for a simple graph on $n$ vertices is the intersection of a lattice and a convex polytope. We show that the set of possible degree sequences for a simple $k$-uniform hypergraph on $n$ vertices is not the intersection of a lattice and a convex polytope for $k \geqslant 3$ and $n \geqslant k+13$. We also show an analogous nonconvexity result for the set of degree sequences of $k$ partite $k$-uniform hypergraphs and the generalized notion of $\lambda$-balanced $k$-uniform hypergraphs.
\end{abstract}

\section{Introduction}

The degree sequence of a graph $G$ on vertices $v_{1}, v_{2}, \ldots, v_{n}$ is the sequence $d(G)=$ $\left(d_{1}, d_{2}, \ldots, d_{n}\right)$, where $d_{i}$ is the degree of the vertex $v_{i}$ in $G$. The Erdös-Gallai Theorem [2] states that a sequence $\left(d_{1}, d_{2}, \ldots, d_{n}\right)$ is the degree sequence of a (simple) graph if and only if $\sum_{i} d_{i}$ is even and the $d_{i}$ satisfy a certain set of inequalities. Koren [4] showed that these inequalities define a convex polytope $D_{n}(2)$, so that the sequences with even sum lying in this polytope are exactly the degree sequences of graphs on $n$ vertices. (For more on this polytope, see [6].)

We consider the analogous question for (simple) $k$-uniform hypergraphs when $k>$ 2. (We assume throughout that all graphs and hypergraphs are simple.) Klivans and Reiner [3] verified computationally that the set of degree sequences for $k$-uniform hypergraphs is the intersection of a lattice and a convex polytope for $k=3$ and $n \leqslant 8$ and asked whether this holds in general. We will show in Section 2 that it does not hold for $k \geqslant 3$ and $n \geqslant k+13$.

Similarly, we can associate to a bipartite graph a pair of degree sequences giving the degrees of the vertices in each part. The Gale-Ryser Theorem [5] gives necessary 
and sufficient conditions in the form of a system of linear inequalities for a pair of degree sequences to arise from a bipartite graph, so that the set of these pairs of degree sequences can again be described as the intersection of a lattice and a convex polytope. We will show in Section 3 that the analogous result does not hold for $k$-partite $k$-uniform hypergraphs if there exist three parts of sizes at least 5, 6, and 6, respectively. We also generalize the notion of $k$-partite $k$-uniform hypergraphs to that of $\lambda$-balanced $k$-uniform hypergraphs and prove a similar statement in this case.

\section{Hypergraph degree sequences}

A (simple) $k$-uniform hypergraph $K$ on the set $[n]=\{1,2, \ldots, n\}$ is a collection of distinct elements (called hyperedges) of $\left(\begin{array}{c}{[n]} \\ k\end{array}\right)$, the $k$-element subsets of $[n]$. The degree sequence of $K$ is $d(K)=\left(d_{1}, d_{2}, \ldots, d_{n}\right)$, where $d_{i}$ is the number of hyperedges in $K$ containing $i$.

We consider degree sequences as points in $\mathbf{R}^{n}$. Let $e_{i}$ be the $i$ th standard basis vector, and for any $S=\left\{i_{1}, \ldots, i_{k}\right\} \subset[n]$, write $e_{S}=e_{i_{1} i_{2} \cdots i_{k}}=e_{i_{1}}+e_{i_{2}}+\cdots+e_{i_{k}}$. Each degree sequence $d(K)$ is the sum of some subset of the $e_{S}$ 's, so the convex hull of all such degree sequences is the zonotope

$$
D=D_{n}(k)=\left\{\sum_{S \in\left(\begin{array}{c}
{[n]} \\
k
\end{array}\right)} c_{S} e_{S} \mid 0 \leqslant c_{S} \leqslant 1\right\} .
$$

(For more on this polytope, see [1].) Moreover, if we let $L \subset \mathbf{Z}^{n}$ be the lattice generated by the $e_{S}$ consisting of lattice points whose coordinates have sum divisible by $k$ (as long as $n>k)$, then each $d(K)$ lies in $D \cap L$. Our main result will be to show that $D \cap L$ contains a point that is not the degree sequence of a $k$-uniform hypergraph when $k \geqslant 3$ and $n \geqslant k+13$.

As a remark, this is closely related to the weaker question of whether every point of $L$ lying in the real cone generated by the $e_{S}$ lies in the semigroup generated by the $e_{S}$. This is well known to be the case and is equivalent to normality of the monomial algebra generated by the $\mathbf{x}^{S}=x_{i_{1}} x_{i_{2}} \cdots x_{i_{k}}$. (See, for instance, [7].) It is also easy to derive the affirmative answer to this question for $\lambda$-balanced hypergraphs as defined in the next section. The essential difference with the present question is that here we are restricted to using each hyperedge at most once.

For a hypergraph $K$, we will define $D(K)$ to be the zonotope generated by the hyperedges in $K$, so

$$
D(K)=\left\{\sum_{S \in K} c_{S} e_{S} \mid 0 \leqslant c_{S} \leqslant 1\right\} .
$$

Lemma 2.1. Let $K$ be a k-uniform hypergraph on $n$ vertices. For any $w \in\left(\mathbf{R}^{*}\right)^{n}$, let $F=F(w)$ be the face of $D(K)$ on which $w$ is maximized. Then $F$ is a translate of $D\left(K^{0}\right)$, where $K^{0}=K^{0}(w) \subset K$ is the set of hyperedges $S \in K$ such that $w\left(e_{S}\right)=0$. Moreover, $F \cap L$ contains a point that is not the degree sequence of a subhypergraph of $K$ if and only if $D\left(K^{0}\right) \cap L$ contains a point that is not the degree sequence of a subhypergraph of $K^{0}$. 
Proof. To maximize $w\left(\sum c_{S} e_{S}\right)=\sum\left(c_{S} \cdot w\left(e_{S}\right)\right)$ for $0 \leqslant c_{S} \leqslant 1$, we must take $c_{S}=1$ when $w\left(e_{S}\right)>0$ and $c_{S}=0$ when $w\left(e_{S}\right)<0$, while $c_{S}$ can be arbitrary if $w\left(e_{S}\right)=0$. Thus $F$ is a translate of $D\left(K^{0}\right)$ by $\sum_{S \in K^{+}} e_{S} \in L$, where $K^{+}$is the set of hyperedges $S$ on which $w$ is positive.

The same argument with the added condition that $c_{S}$ equals either 0 or 1 for all $S$ shows that the degree sequences in $F$ are exactly the translations of degree sequences of subhypergraphs of $K^{0}$.

Therefore, to find a point of $D \cap L$ that is not the degree sequence of a $k$-uniform hypergraph, it suffices, by Lemma 2.1 , to take $K=\left(\begin{array}{c}{[n]} \\ k\end{array}\right)$ and to exhibit a weight vector $w$ and a point of $D\left(K^{0}\right) \cap L$ that is not the degree sequence of a subhypergraph of $K^{0}$.

Proposition 2.2. Let $k=3$ and $n=16$. If

$$
w=(8,6,6,4,1,1,0,0,0,0,-2,-2,-3,-3,-5,-12)
$$

then

$$
p=(2,1,1,2,1,1,1,1,1,1,1,1,2,2,2,1)
$$

lies in $D\left(K^{0}\right) \cap L$ but is not the degree sequence of a subhypergraph of $K^{0}$.

Proof. Since the sum of the entries of $p$ is $21=3 \cdot 7, p$ lies in $L$. Also,

$$
\begin{aligned}
p=\frac{1}{3}\left(e_{2,3,16}+e_{4,5,15}+e_{4,6,15}\right. & \left.+e_{5,6,11}+e_{5,6,12}+e_{7,8,9}+e_{7,8,10}+e_{7,9,10}+e_{8,9,10}\right) \\
+ & \frac{2}{3}\left(e_{1,4,16}+e_{1,13,15}+e_{1,14,15}+e_{2,13,14}+e_{3,13,14}+e_{4,11,12}\right) .
\end{aligned}
$$

Since $w$ vanishes on each $e_{S}$ on the right side, it follows that $p \in D\left(K^{0}\right)$. However, $p$ is not the degree sequence of a subhypergraph of $K^{0}$ : since $w_{7}=w_{8}=w_{9}=w_{10}=0$ but otherwise $w_{i} \neq 0$ and $w_{i} \neq-w_{j}$ for distinct $i, j \notin\{7,8,9,10\}$, we have $\left(e_{7}+e_{8}+e_{9}+\right.$ $\left.e_{10}\right) \cdot e_{S}$ is 0 or 3 for any $S \in K^{0}$ (where - indicates the usual inner product on $\mathbf{R}^{n}$ ). But $\left(e_{7}+e_{8}+e_{9}+e_{10}\right) \cdot p=4$, which is not divisible by 3 , so $p$ cannot be the sum of some $e_{S}$ for $S \in K^{0}$.

Using this, we can easily derive the following.

Theorem 2.3. For $k \geqslant 3$ and $n \geqslant k+13$, the set of degree sequences of $k$-uniform hypergraphs on $n$ vertices is not the intersection of a lattice and a convex polytope.

Proof. It suffices to show that there is a point in $D \cap L$ that is not a degree sequence (since $D$ and $L$ are the smallest convex polytope and lattice containing all degree sequences). Combining Lemma 2.1 and Proposition 2.2 gives the result for $k=3$ and $n=16$. Since $D_{n}(k)$ is the face of $D_{n+1}(k)$ with last coordinate 0 , Lemma 2.1 also gives the result for $k=3$ and $n \geqslant 16$.

Consider the map $f:\left(d_{1}, d_{2}, \ldots, d_{n}\right) \mapsto\left(d_{1}, d_{2}, \ldots, d_{n}, \frac{1}{k}\left(d_{1}+\cdots+d_{n}\right)\right)$. If $d$ is a $k$-uniform hypergraph degree sequence on $n$ vertices, then $f(d)$ is a $(k+1)$-uniform 
hypergraph degree sequence on $n+1$ vertices (simply add vertex $n+1$ to all hyperedges). Conversely, if the degree sequence of a $(k+1)$-uniform hypergraph satisfies $d_{n+1}=\frac{1}{k}\left(d_{1}+\cdots+d_{n}\right)$, then every hyperedge must contain the vertex $n+1$, so it is of the form $f(d)$ for some $k$-uniform hypergraph degree sequence $d$. Since $f$ is linear, it also sends $D_{n}(k)$ into $D_{n+1}(k+1)$, so any counterexample for $(n, k)$ yields a counterexample for $(n+1, k+1)$. An easy induction completes the proof.

It is possible that with additional work or computation the constant 13 may be improved.

In the next section, we will prove an analogous result for $k$-partite $k$-uniform hypergraphs as well as the more general $\lambda$-balanced hypergraphs. (Our construction below can also be used to prove Theorem 2.3 but with a constant of 14 instead of 13.)

\section{$3 \quad \lambda$-balanced hypergraphs}

Let $\lambda=\left(\lambda_{1}, \lambda_{2}, \ldots, \lambda_{p}\right)$ be a partition of $k$. We say a $k$-uniform hypergraph is $\lambda$-balanced if its vertex set can be partitioned into $p$ sets $V_{1}, \ldots, V_{p}$ such that each hyperedge contains $\lambda_{i}$ vertices from $V_{i}$. (We will also call a hyperedge $\lambda$-balanced if it satisfies this property.) A $(1,1, \ldots, 1)$-balanced $k$-uniform hypergraph is called $k$-partite. Note that every $k$-uniform hypergraph is $(k)$-balanced.

Let $n_{i}=\left|V_{i}\right|$, and label the vertices in $V_{i}$ by $v_{1}^{i}, v_{2}^{i}, \ldots, v_{n_{i}}^{i}$. We then associate to a $\lambda$-balanced hypergraph $K$ a degree sequence

$$
d=\left(d_{1}^{1}, d_{2}^{1}, d_{3}^{1}, \ldots ; \quad d_{1}^{2}, d_{2}^{2}, \ldots ; \quad \ldots ; \quad d_{1}^{p}, d_{2}^{p}, \ldots\right),
$$

where $d_{j}^{i}$ gives the number of hyperedges in $K$ containing vertex $v_{j}^{i}$. As before, this degree sequence is $\sum_{S \in K} e_{S}$, where $e_{S}$ is the sum of the standard basis vectors in $\mathbf{R}^{n_{1}} \times \mathbf{R}^{n_{2}} \times$ $\cdots \times \mathbf{R}^{n_{p}}$ corresponding to vertices in the hyperedge $S$. When $n_{i}>\lambda_{i}$ for all $i$, the lattice $L$ generated by all possible $e_{S}$ consists of all sequences $d$ for which there exists $q \in \mathbf{Z}$ such that $\sum_{j=1}^{n_{i}} d_{j}^{i}=\lambda_{i} q$ for all $i$. (In other words, the sum of the degrees of the vertices in $V_{i}$ must be the same integer multiple of $\lambda_{i}$.)

As before, we let $D$ be the zonotope generated by all $e_{S}$ for $\lambda$-balanced hyperedges $S$ and ask whether all points in $D \cap L$ are degree sequences for $\lambda$-balanced hypergraphs. We will again find that this is not the case for any $\lambda$ when $k \geqslant 3$ and the $n_{i}$ are sufficiently large. We first consider a special case.

Proposition 3.1. Let $\lambda=(1,1,1)$ and $\left(n_{1}, n_{2}, n_{3}\right)=(5,6,6)$. Also let

$$
w=(-7,-7,-7,-7,-7 ; \quad 1,1,2,2,3,3 ; \quad 6,6,5,5,4,4),
$$

and define $K^{0}$ as in Lemma 2.1. Then

$$
p=(11,9,6,3,1 ; \quad 2,4,6,8,3,7 ; \quad 2,4,6,8,3,7)
$$

lies in $D\left(K^{0}\right) \cap L$ but is not the degree sequence of a subhypergraph of $K^{0}$. 
Proof. Define points

$$
\begin{array}{lll}
p^{-}=(10,8,4,2,0 ; & 1,3,5,7,2,6 ; & 1,3,5,7,2,6), \\
p^{+}=(12,10,8,4,2 ; & 3,5,7,9,4,8 ; & 3,5,7,9,4,8),
\end{array}
$$

so $p=\frac{1}{2}\left(p^{-}+p^{+}\right)$. Note that the sum of the coordinates of the three parts of $p^{-}$are all 24 , so $p^{-} \in L$. Likewise, $p^{+}$and $p$ also lie in $L$.

Let

$$
A=\left(a_{r s}\right)=\left(\begin{array}{llllll}
1 & 2 & 0 & 0 & 0 & 0 \\
2 & 3 & 0 & 0 & 0 & 0 \\
0 & 0 & 3 & 4 & 0 & 0 \\
0 & 0 & 4 & 5 & 0 & 0 \\
0 & 0 & 0 & 0 & 1 & 3 \\
0 & 0 & 0 & 0 & 3 & 5
\end{array}\right)
$$

Note that $K^{0}=\left\{\left\{v_{q}^{1}, v_{r}^{2}, v_{s}^{3}\right\} \mid 1 \leqslant q \leqslant 5,1 \leqslant r, s \leqslant 6, a_{r s} \neq 0\right\}$.

Then $p^{-}=\sum e_{S}$, where the sum ranges over all $S=\left\{v_{q}^{1}, v_{r}^{2}, v_{s}^{3}\right\}$ such that $q<a_{r s}$. Likewise $p^{+}=\sum e_{S}$, where the sum now ranges over all $S=\left\{v_{q}^{1}, v_{r}^{2}, v_{s}^{3}\right\}$ with $q \leqslant a_{r s}$. Therefore $p^{-}, p^{+}$, and their midpoint $p$ lie in $D\left(K^{0}\right)$.

We will now show that $p$ is not the degree sequence of a hypergraph that uses only hyperedges in $K^{0}$. Suppose it were, so that we could write $p=\sum_{S \in K} e_{S}$ for some $K \subset K^{0}$. Let $B=\left(b_{r s}\right)$ be the $6 \times 6$ matrix such that $b_{r s}$ counts the number of $q$ for which $\left\{v_{q}^{1}, v_{r}^{2}, v_{s}^{3}\right\} \in K$. Then the sequence of row and column sums of $B$ must both be $(2,4,6,8,3,7)$. Since we also know that $0 \leqslant b_{r s} \leqslant 5$, this means that:

$$
\begin{aligned}
& B_{1}=\left(\begin{array}{ll}
b_{11} & b_{12} \\
b_{21} & b_{22}
\end{array}\right) \in\left\{\left(\begin{array}{ll}
0 & 2 \\
2 & 2
\end{array}\right),\left(\begin{array}{ll}
1 & 1 \\
1 & 3
\end{array}\right),\left(\begin{array}{ll}
2 & 0 \\
0 & 4
\end{array}\right)\right\}, \\
& B_{2}=\left(\begin{array}{ll}
b_{33} & b_{34} \\
b_{43} & b_{44}
\end{array}\right) \in\left\{\left(\begin{array}{ll}
1 & 5 \\
5 & 3
\end{array}\right),\left(\begin{array}{ll}
2 & 4 \\
4 & 4
\end{array}\right),\left(\begin{array}{ll}
3 & 3 \\
3 & 5
\end{array}\right)\right\}, \\
& B_{3}=\left(\begin{array}{ll}
b_{55} & b_{56} \\
b_{65} & b_{66}
\end{array}\right) \in\left\{\left(\begin{array}{ll}
0 & 3 \\
3 & 4
\end{array}\right),\left(\begin{array}{ll}
1 & 2 \\
2 & 5
\end{array}\right)\right\} .
\end{aligned}
$$

Moreover, for $1 \leqslant r, s \leqslant 6$, the pair $\left\{v_{r}^{2}, v_{s}^{3}\right\}$ can appear in at most $\min \left\{q, b_{r s}\right\}$ hyperedges with one of the vertices in $\left\{v_{1}^{1}, \ldots, v_{q}^{1}\right\}$. Therefore, if we let $\mu=(11,9,6,3,1)$, then $\mu_{1}+\cdots+\mu_{q} \leqslant \sum_{r, s} \min \left\{q, b_{r s}\right\}$. In other words, if $\nu=\left(\nu_{1}, \ldots, \nu_{5}\right)$ is the partition such that $\nu_{q}$ counts the number of $b_{r s}$ that are at least $q$, then $\mu_{1}+\cdots+\mu_{q} \leqslant \nu_{1}+\cdots+\nu_{q}$.

It is now straightforward to show that there are no possible choices of $B_{1}, B_{2}$, and $B_{3}$ satisfying these conditions: if $B_{3}=\left(\begin{array}{ll}0 & 3 \\ 3 & 4\end{array}\right)$, we cannot choose $B_{1}$ such that both $\mu_{1} \leqslant \nu_{1}$ and $\mu_{1}+\mu_{2} \leqslant \nu_{1}+\nu_{2}$. Similarly if $B_{3}=\left(\begin{array}{ll}1 & 2 \\ 2 & 5\end{array}\right)$, we cannot choose $B_{2}$ such that both $\mu_{1}+\mu_{2}+\mu_{3} \leqslant \nu_{1}+\nu_{2}+\nu_{3}$ and $\mu_{1}+\mu_{2}+\mu_{3}+\mu_{4} \leqslant \nu_{1}+\nu_{2}+\nu_{3}+\nu_{4}$. Thus $p \in D\left(K^{0}\right) \cap L$ is not the degree sequence of a hypergraph using only hyperedges in $K^{0}$.

Combining Lemma 2.1 and Proposition 3.1 gives our desired result for 3-partite 3uniform hypergraphs, and we can easily extend this result to $k$-partite $k$-uniform hypergraphs. 
Theorem 3.2. For $k \geqslant 3$, consider $k$-partite $k$-uniform hypergraphs with parts of sizes $n_{1}, n_{2}, \ldots, n_{k}$ for which $n_{1} \geqslant 5, n_{2} \geqslant 6, n_{3} \geqslant 6$, and $n_{i} \geqslant 1$ otherwise. The corresponding set of degree sequences is not the intersection of a lattice and a convex polytope.

Proof. As in Theorem 2.3, combining Lemma 2.1 and Proposition 3.1 gives the result for $k=3$ and $\left(n_{1}, n_{2}, n_{3}\right)=(5,6,6)$. Also note that the polytopes and lattices for $k \geqslant 3$ with $\left(n_{1}, n_{2}, n_{3}, n_{4}, \ldots, n_{k}\right)=(5,6,6,1, \ldots, 1)$ are all identical to the $k=3$ case (by projecting away the last $k-3$ coordinates) so this also proves those cases. Finally, increasing any $n_{i}$ but restricting to the face of the zonotope where the new vertices have degree 0 again reduces to the same case by Lemma 2.1, completing the proof.

Theorem 3.2 is also easy to extend to $\lambda$-balanced hypergraphs for all $\lambda$ when $k \geqslant 3$. Consider a $\lambda$-balanced hypergraph on vertex sets $V_{1}, \ldots, V_{p}$ of sizes $n_{1}, n_{2}, \ldots, n_{p}$. We will say that $\left(n_{1}, \ldots, n_{p}\right)$ is a $\lambda$-coarsening of $\left(m_{1}, \ldots, m_{k}\right)$ if each $V_{i}$ can be partitioned into $\lambda_{i}$ sets such that the sizes of all the resulting sets are $m_{1}, \ldots, m_{k}$.

Theorem 3.3. Consider $\lambda$-balanced hypergraphs with parts of sizes $n_{1}, \ldots, n_{p}$, where $\left(n_{1}, \ldots, n_{p}\right)$ is a $\lambda$-coarsening of $\left(m_{1}, m_{2}, \ldots, m_{k}\right)$ such that Theorem 3.2 holds for parts of sizes $m_{1}, \ldots, m_{k}$. (In particular, this will hold whenever the $n_{i}$ are sufficiently large.) Then the corresponding set of degree sequences is not the intersection of a lattice and a convex polytope.

Proof. Let the vertex sets $V_{1}, \ldots, V_{p}$ have corresponding coarsening $W_{1}, \ldots, W_{k}$. It suffices to exhibit a weight vector $w$ such that the corresponding $K^{0}$ as in Lemma 2.1 is the complete $k$-partite $k$-uniform hypergraph on $W_{1}, \ldots, W_{k}$. Indeed, any hyperedge in $K^{0}$ will be $\lambda$-balanced by the definition of $\lambda$-coarsening, and the lattice generated by hyperedges in $K^{0}$ is a sublattice of the lattice generated by all $\lambda$-balanced hyperedges. Therefore any counterexample for $K^{0}$ will yield a counterexample for $\lambda$-balanced hypergraphs as in Lemma 2.1.

To exhibit such a weight vector, let $N$ be an integer larger than any $m_{i}$. Then let the weight of vertices in $W_{1}$ be $-\left(1+N+N^{2}+\cdots+N^{k-2}\right)$ and in $W_{i}$ be $N^{i-2}$ for $2 \leqslant i \leqslant k$. Then the only way to pick $k$ vertices the sum of whose weights is 0 is to take one from each $W_{i}$. In other words, the only hyperedges in $K^{0}$ are those that have one vertex from each $W_{i}$, as desired.

\section{Acknowledgments}

The author would like to thank Victor Reiner for suggesting this direction of study, as well as for useful discussions and overall encouragement. This work was supported by a National Science Foundation Mathematical Sciences Postdoctoral Research Fellowship. 


\section{References}

[1] N. L. Bhanu Murthy and Murali K. Srinivasan. The polytope of degree sequences of hypergraphs. Linear Algebra Appl., 350:147-170, 2002.

[2] Paul Erdős and Tibor Gallai. Graphs with prescribed degrees of vertices (in Hungarian). Matematikai Lopak, 11:264-274, 1961.

[3] C. Klivans and V. Reiner. Shifted set families, degree sequences, and plethysm. Electron. J. Combin., 15(1):Research Paper 14, 35, 2008.

[4] Michael Koren. Extreme degree sequences of simple graphs. J. Combinatorial Theory Ser. B, 15:213-224, 1973.

[5] Herbert John Ryser. Combinatorial mathematics. The Carus Mathematical Monographs, No. 14. Published by The Mathematical Association of America, 1963.

[6] Richard P. Stanley. A zonotope associated with graphical degree sequences. In Applied geometry and discrete mathematics, volume 4 of DIMACS Ser. Discrete Math. Theoret. Comput. Sci., pages 555-570. Amer. Math. Soc., Providence, RI, 1991.

[7] Bernd Sturmfels. Gröbner bases and convex polytopes, volume 8 of University Lecture Series. American Mathematical Society, Providence, RI, 1996. 